\title{
COVID-19 in patients with Down syndrome
}

\author{
Amir Emami $^{1} \cdot$ Fatemeh Javanmardi $^{1} \cdot$ Ali Akbari $^{2} \cdot$ Ali A. Asadi-Pooya ${ }^{3,4}$
}

Received: 31 December 2020 / Accepted: 23 January 2021 / Published online: 1 February 2021

(C) Fondazione Società Italiana di Neurologia 2021

\begin{abstract}
Purpose The aim of the current study was to determine whether COVID-19 is associated with a different presenting clinical picture or a more severe course of illness in people with Down syndrome (DS).

Methods All consecutive patients who were admitted at healthcare facilities anywhere in Fars province (located in the south of Iran with a population of 4,851,000 people) from 19 February 2020 to 20 November 2020 were included. For every patient with DS, three age- and sex-matched patients with COVID-19 and without any underlying medical conditions were selected as controls.

Results During the study period, 37,968 patients were hospitalized with a diagnosis of COVID-19. Eighteen patients had DS. Patients with DS were significantly more likely to be intubated [7 patients (39\%)] compared with those without DS [3 patients $(6 \%)] ; p=0.002$. Patients with DS significantly more often died of COVID-19 compared with the controls [8 (44.4\%) vs. 1 (1.9\%); odds ratio: 24.37 ; $95 \%$ confidence interval $2.39-247.94 ; p=0.007]$.

Conclusion Patients with DS are among the high-risk populations with respect to severe COVID-19 and should receive the vaccine as soon as possible. Furthermore, they should receive more intensive care if they get hospitalized with the illness.
\end{abstract}

Keywords Coronavirus · COVID · Down syndrome

\section{Introduction}

Severe acute respiratory syndrome (SARS) coronavirus-2 (SARS-CoV-2) has caused a catastrophic pandemic since late

Ali A. Asadi-Pooya

aliasadipooya@yahoo.com

Amir Emami

Emami.microbia@gmail.com

Fatemeh Javanmardi

javanmardi.biostat@yahoo.com

Ali Akbari

aeshahin2002@yahoo.com

1 Burn \& Wound Healing Research Center, Department of Microbiology, Shiraz University of Medical Sciences, Shiraz, Iran

2 Department of Anesthesiology, School of Medicine, Shiraz University of Medical Sciences, Shiraz, Iran

3 Epilepsy Research Center, Shiraz University of Medical Sciences, Shiraz, Iran

4 Jefferson Comprehensive Epilepsy Center, Department of Neurology, Thomas Jefferson University, Philadelphia, PA, USA
2019 (COVID-19) [1]. COVID-19 is primarily a respiratory illness, but patients may experience a severe illness characterized by multi-organ failure and death [1]. The main risk factors for a more severe COVID-19 have been recognized as older age, diabetes mellitus, chronic respiratory problems, hypertension, and cardiovascular disorders, among others $[1,2]$.

Recently, a handful of COVID-19 vaccines have received approval from the authorities or are in the pipeline [3]. However, it will be a long process before everyone in the world can get vaccinated against this fatal illness. Even in wealthy countries, the governments should make a plan to prioritize the people who need the vaccine most [4]. Variables such as occupational exposure, age, and underlying health conditions may help policymakers to prepare a fair plan in order to prioritize the people, who need the COVID-19 vaccine most [4].

Down syndrome (DS) is a genetic disorder with several congenital defects (e.g., cardiac, respiratory, immunological) [5]. People with DS have a higher risk of being hospitalized with viral respiratory infections (e.g., respiratory syncytial virus and influenza virus) [6,7]. Likelihoods of intubation and death were 8-fold and 335-fold greater, respectively, for patients with DS compared with others during the H1N1 
pandemic 2009 in Mexico [7]. Therefore, it is reasonable to hypothesize that DS is a major risk factor for severe and fatal COVID-19.

The aim of the current study was to determine whether COVID-19 is associated with a different presenting clinical picture or a more severe course of illness (e.g., intubation and death) in people with DS; this information might help us understand the severity and the gravity of COVID-19 in people with DS.

\section{Methods}

\section{Patients}

Iran reported its first confirmed cases of COVID-19 on 19 February 2020. As of 9 December 2020, there have been 50,917 COVID-19-related deaths with 1,062,397 confirmed infections in Iran [8]. In this study, all consecutive patients who were referred to and were admitted at healthcare facilities anywhere in Fars province (located in the south of Iran with a population of 4,850,000 people) from 19 February 2020 to 20 November 2020 were included. The patients had a confirmed COVID-19 diagnosis by a positive result on real-time polymerase chain reaction (qRT-PCR) testing of nasopharyngeal and oropharyngeal samples, or a probable COVID-19 diagnosis by a positive chest computerized scan (CT) characteristic for COVID-19, or a possible COVID-19 diagnosis by clinical manifestations (i.e., history and physical exam) compatible with COVID-19.

\section{Data collection}

For any hospitalized patient, the following data were collected and entered into a database (on admission): age, sex, presence of fever, cough, respiratory distress, muscle pain, loss of smell, dizziness, headache, seizure, abdominal pain, nausea, vomiting, and anorexia. Other collected data included the following: qRT-PCR test results, chest CT scan results, and the need for intubation. Underlying chronic health problems were also collected (self-declared or through the caregivers when the patient's mental status was impaired) [i.e., renal, liver, cardiac, and also diabetes mellitus (DM), cancer, hypertension (HTN), down syndrome, asthma). No data were available about the hospital course of the patients (e.g., laboratory test results, complications). However, the outcome was also recorded in the database as dead or discharged.

\section{Statistical analyses}

For every patient with DS, three age- and sex-matched patients with COVID-19 and without any underlying medical conditions were selected as controls. Values were presented as mean \pm standard deviation (SD) for continuous variables and as number (percent) of subjects for categorical variables. Fisher's exact test and $t$ test were used for univariate comparisons. Bonferroni correction test was used. The associations between DS and other variables were determined using the logistic regression analysis; the significant variables from the univariate analyses were entered into the logistic regression analysis model. Odds ratios (ORs) and 95\% confidence intervals (CIs) were calculated. A $p$ value less than 0.05 (twosided) was considered significant.

\section{Standard protocol approvals, registrations, and patient consents}

Shiraz University of Medical Sciences Review Board approved this study as a minimal-risk research using the data that were collected for routine clinical practice and waived the requirement for informed consent. Information was collected and entered into the electronic registry of Shiraz University of Medical Sciences (Ethics code: IR.SUMS.REC.1399.022).

\section{Data availability statement}

The data are confidential and will not be shared as per the regulations of Shiraz University of Medical Sciences.

\section{Results}

\section{General characteristics of the patients}

During the study period, 37,968 patients [20,189 (53.2\%) men and $17,779(46.8 \%)$ women] were hospitalized with a diagnosis of COVID-19 [13,165 patients (34.7\%) with confirmed diagnoses, 9400 patients $(24.8 \%)$ with probable diagnoses, and 15,403 patients $(40.6 \%)$ with possible diagnoses]. The mean age ( \pm standard deviation) of the patients was $52( \pm$ 23) years (range 0 to 129 years). Eighteen patients had DS and were hospitalized with COVID-19. Three patients with DS had cardiac problems, one had diabetes mellitus, and one had cardio-pulmonary problems. Fifty-four age- and sexmatched patients with COVID-19 and without any underlying medical conditions were selected as controls. The distribution of the confirmed/probable/possible COVID-19 diagnoses was similar between the groups (7/3/8 in the DS group and 17/5/32 in the control group; $p=0.49$ ).

\section{Presenting clinical manifestations of COVID-19 in people with DS}

Table 1 shows the presenting clinical manifestations of COVID-19 in people with DS compared with those in 
Table 1 Clinical characteristics of patients with COVID-19 with or without Down syndrome in univariate analyses

\begin{tabular}{|c|c|c|c|}
\hline Variable (statistical test) & Patients with Down syndrome, $N=18(\%)$ & Controls, $N=54(\%)$ & $p$ value \\
\hline Age (mean \pm standard deviation), years $(t)$ & $\begin{array}{l}28.6 \pm 14.5 \text { (range } 1 \text { to } 54 \text { years; } \\
\quad \text { median } 30 ; \text { interquartile range } 21)\end{array}$ & $\begin{array}{l}28.0 \pm 12.6 \text { (range } 1 \text { to } 54 \text { years; } \\
\quad \text { median } 30 ; \text { interquartile range } 13 \text { ) }\end{array}$ & 0.868 \\
\hline Sex (male: female) (FE) & $11: 7$ & $33: 21$ & 1.000 \\
\hline Fever (FE) & $8(44 \%)$ & $22(41 \%)$ & 0.790 \\
\hline Cough (FE) & $7(39 \%)$ & $24(44 \%)$ & 0.787 \\
\hline Muscle pain (FE) & $5(28 \%)$ & $18(33 \%)$ & 0.775 \\
\hline Respiratory distress (FE) & $11(61 \%)$ & $17(31 \%)$ & 0.049 \\
\hline Loss of smell (FE) & 0 & $4(7 \%)$ & 0.566 \\
\hline Headache (FE) & 0 & $12(22 \%)$ & 0.030 \\
\hline Dizziness (FE) & 0 & $2(4 \%)$ & 1.000 \\
\hline Seizure (FE) & 0 & 0 & - \\
\hline Abdominal pain (FE) & 0 & $1(2 \%)$ & 1.000 \\
\hline Nausea (FE) & 0 & $3(5 \%)$ & 0.568 \\
\hline Vomiting (FE) & $2(11 \%)$ & $5(9 \%)$ & 1.000 \\
\hline Anorexia (FE) & 0 & $2(4 \%)$ & 1.000 \\
\hline Diarrhea (FE) & $1(6 \%)$ & $4(7 \%)$ & 1.000 \\
\hline Intubation (FE) & $7(39 \%)$ & $3(6 \%)$ & 0.002 \\
\hline Death (FE) & $8(44.4 \%)$ & $1(1.9 \%)$ & 0.0001 \\
\hline
\end{tabular}

After Bonferroni correction test, a significant $p$ value is 0.003

$t t$ test, $F E$ Fisher's exact test

controls. Both groups had similar presenting clinical manifestations. Neurological manifestations (i.e., loss of smell, headache, dizziness, seizure) were not among the presenting manifestations of COVID-19 in patients with DS.

\section{Clinical course and outcome of COVID-19 in people with DS}

Patients with DS were significantly more likely to be intubated. They were also more likely to die of COVID-19 during their hospital admission compared with the controls (Table 1). We included these two variables in a regression analysis model. The model that was generated by this test was significant $(p=0.0001)$. Patients with DS significantly more often died of COVID-19 compared with the controls [OR 24.37; 95\%CI 2.39-247.94; $p=0.007$ ]. Intubation lost its significance $(p=0.236)$.

\section{Discussion}

In the current study, we observed that people with DS are significantly at risk of dying if they get hospitalized with COVID-19 compared with people without any underlying health problems and COVID-19. In a previous study, the relative risk of mortality among hospitalized patients with DS and COVID-19 was 2.9 compared with that in controls [9]. Another study of 12 patients with DS and
COVID-19 showed that individuals with DS, who were hospitalized with COVID-19, had a more severe disease than age-matched controls [10]. These three studies identify people with DS as a high-risk population for severe COVID-19 with a grave prognosis.

Down syndrome (trisomy 21) is the most common chromosomal abnormality in humans. These patients suffer from an immune dysregulation that predisposes them to autoimmune disorders. These patients also have other congenital anomalies (e.g., cardiac, respiratory tract) that predispose them to a high frequency of severe respiratory diseases, the leading cause of mortality in people with DS $[11,12]$. Considering these data, policymakers should prioritize the people with DS to receiving the COVID-19 vaccine, irrespective of their age.

In the current study, we also observed that patients with DS and COVID-19 had similar presenting clinical manifestations compared with age- and sex-matched people with COVID-19 and without any underlying medical conditions. Similar to the control group, the most frequent signs and symptoms of COVID-19 in people with DS were respiratory distress, fever, cough, and muscle pain. A previous study corroborates this observation [9]. Since we did not have access to the hospital course and the full clinical picture of the studied patients, we cannot comment on those issues here. However, a previous study showed that patients with DS had a more severe disease than controls, with particularly an increased incidence of sepsis and chance of mechanical ventilation [10]. 


\section{Limitations}

We did not have access to the whole medical records of the patients to clarify the full clinical picture of these patients. Furthermore, we only studied the hospitalized patients; the results could be different in the general population (including the asymptomatic and mild cases of COVID-19).

\section{Conclusion}

Patients with DS are among the high-risk populations with respect to severe COVID-19 and should receive the vaccine as soon as possible. Furthermore, they should receive more intensive attention and care if they get hospitalized with the illness.

Acknowledgements Shiraz University and Shiraz University of Medical Sciences supported this study.

Author contribution Ali A. Asadi-Pooya, M.D.: design and conceptualized the study, analyzed the data, and drafted and revised the manuscript.

Others: data collection and revised the manuscript.

Funding Shiraz University and Shiraz University of Medical Sciences had no role in the study design; in the collection, analysis, and interpretation of the data; in the writing of the report; and in the decision to submit the paper for publication.

Availability of data and materials The data used in this study are confidential and will not be shared.

\section{Declarations}

Ethical approval None

Conflict of interest Ali A. Asadi-Pooya, M.D.: Honoraria from Cobel Daruo, RaymandRad, Sanofi, and Tekaje; Royalty: Oxford University Press (Book publication). Others: none.

\section{References}

1. Rothan HA, Byrareddy SN (2020) The epidemiology and pathogenesis of coronavirus disease (COVID-19) outbreak. J Autoimmun 109:102433

2. Javanmardi F, Keshavarzi A, Akbari A, Emami A, Pirbonyeh N (2020) Prevalence of underlying diseases in died cases of COVID19: a systematic review and meta-analysis. PLoS One 15:e0241265

3. https://www.who.int/emergencies/diseases/novel-coronavirus2019/covid-19-vaccines/. Accessed on 21 January 2020

4. https://www.gov.uk/government/publications/priority-groups-forcoronavirus-covid-19-vaccination-advice-from-the-jcvi-2december-2020/. Accessed on December 9, 2020

5. Altable M, de la Serna JM (2020) Down's syndrome and COVID19: risk or protection factor against infection? A molecular and genetic approach. Neurol Sci 42:407-413. https://doi.org/10.1007/ s10072-020-04880-x

6. Zachariah P, Ruttenber M, Simões EA (2012) Down syndrome and hospitalizations due to respiratory syncytial virus: a populationbased study. J Pediatr 160:827-831

7. Pérez-Padilla R, Fernández R, García-Sancho C, Franco-Marina F, Aburto O, López-Gatell H, Bojórquez I (2010) Pandemic (H1N1) 2009 virus and Down syndrome patients. Emerg Infect Dis 16: $1312-1314$

8. https://www.worldometers.info/coronavirus/country/iran/. Accessed on December 9, 2020

9. Hüls A, Costa ACS, Dierssen M et al (2020) An international survey on the impact of COVID-19 in individuals with Down syndrome. medRxiv:2020.11.03.20225359. https://doi.org/10.1101/ 2020.11.03.20225359 Preprint

10. Malle L, Gao C, Hur C et al (2020) Individuals with Down syndrome hospitalized with COVID-19 have more severe disease. Genet Med. https://doi.org/10.1038/s41436-020-01004-w Online ahead of print

11. Englund A, Jonsson B, Zander CS, Gustafsson J, Annerén G (2013) Changes in mortality and causes of death in the Swedish Down syndrome population. Am J Med Genet A 161A:642-649

12. Vita S, Di Bari V, Corpolongo A et al (2020) Down syndrome patients with COVID-19 pneumonia: a high-risk category for unfavourable outcome. Int J Infect Dis S1201-9712(20):3250332500

Publisher's note Springer Nature remains neutral with regard to jurisdictional claims in published maps and institutional affiliations. 